\title{
Vein Disorder
}

National Cancer Institute

\section{Source}

National Cancer Institute. Vein Disorder. NCI Thesaurus. Code C35279.

Any disorder of the veins. 